\title{
Encoding True Second-order Arithmetic in the Real-Algebraic Structure of Models of Intuitionistic Elementary Analysis
}

(Draft)

\section{Miklós Erdélyi-Szabó}

Alfréd Rényi Institute of Mathematics

Hungary

1053 Budapest, Reáltanoda u. 13-15

\begin{abstract}
Based on the paper [1] we show that true second-order arithmetic is interpretable over the real-algebraic structure of models of intuitionistic analysis built upon a certain class of complete Heyting algebras.
\end{abstract}

Mathematics Subject Classification: 03-D35, 03-F55.

Keywords: Undecidability, Intuitionism, Heyting algebra, True second-order arithmetic

\section{Introduction}

Let $L$ denote the language of ordered rings. In [2] we showed the undecidability of the $L$-structure of Scott's model (see [3]). Continuing the investigation, in [1], we showed that true first-order arithmetic is interpretable in the $L$-structure of a class of models which includes the well-known topological models as well as Scowcroft's model (defined in [4]) and its generalizations. Here we improve that result showing the interpretability of true second-order arithmetic in these structures. We shall use the notations, definitions and the results of that earlier paper.

\section{Basic notions}

We quote the main (standard) definitions about the models we are interested in from [1].

\footnotetext{
${ }^{1}$ e-mail: mszabo.at.math.renyi.hu
} 
Let $\mathcal{H}=(H, \perp, \top, \wedge, \vee, \rightarrow, \leq)$ be a complete Heyting algebra - the truth value algebra of the model to be defined. Infinite infimum and supremum will be denoted by $\bigwedge$ and $\bigvee$ respectively. We say that $A \in H$ is complemented if there is an element denoted by $A^{c}$ of $H$ such that $A \wedge A^{c}=\perp$ and $A \vee A^{c}=\top$. Note that if $\mathrm{A}$ is complemented then $A^{c}=A \rightarrow \perp . A \rightarrow \perp$ is the pseudocomplement of $A$, denoted by $\neg A$. We say that two elements $U_{1}, U_{2} \in H$ are disjoint if $U_{1} \wedge U_{2}=\perp$. An element $U \in H$ is dense if $\neg \neg U=\top$.

A choice sequence, i.e. a sequence of natural numbers is represented in the model by a function $\xi: \omega \times \omega \rightarrow H$ such that $\bigvee_{m} \xi(l, m)=\top$ and $\xi(l, m) \wedge \xi(l, n)=\perp$ for all $l, m, n$ with $m \neq n$. From this follows that the elements $\xi(l, m)$ are complemented. Here $\xi(l, m)$ is the truth value of the statement that the $l$-th element of the sequence represented by $\xi$ is $m$. Let $\Xi$ denote the set of choice sequences.

The language $L_{1}$ we shall use is the one used in [4]. It contains two sorts of variables - $x, y, z$, etc. ranging over the elements of $\omega$, and $\alpha, \beta$, etc. ranging over choice sequences - and constant symbols for each $m \in \omega$ and for each choice sequence $\xi$. Since it will not cause any confusion, we shall use the same symbol for the constant and the corresponding element of the model. The language contains symbols for certain primitive recursive functions and relations defined on the elements of $\omega$ - e.g. $|x-y|, \leq$ etc. - and we also have the equality symbol $=$. It will be used in atomic formulas of the form $t=t^{\prime}$ or $\xi(t)=t^{\prime}$ where $t$ and $t^{\prime}$ are terms of natural-number sort and $\xi$ is a choice sequence (constant).

Atomic sentences receive truth values as follows:

1. $\|m \leq n\|= \begin{cases}\top & \text { if } m \leq n \\ \perp & \text { if } m \not \leq n\end{cases}$

and similarly for other primitive-recursive relations.

2. Concerning choice sequences, we have:

$\|\xi(m)=n\|=\xi(m, n)$.

Arbitrary sentences $\varphi$ receive truth values $\|\varphi\| \in H$ in the usual way:

1. $\left\|\varphi_{1} \vee \varphi_{2}\right\|=\left\|\varphi_{1}\right\| \vee\left\|\varphi_{2}\right\|$

2. $\left\|\varphi_{1} \wedge \varphi_{2}\right\|=\left\|\varphi_{1}\right\| \wedge\left\|\varphi_{2}\right\|$

3. $\left\|\varphi_{1} \rightarrow \varphi_{2}\right\|=\left\|\varphi_{1}\right\| \rightarrow\left\|\varphi_{2}\right\|$ 
4. $\|\neg \varphi\|=\|\varphi\| \rightarrow \perp$

5. $\|\exists x \varphi(x)\|=\bigvee_{n \in \omega}\|\varphi(n)\|$

6. $\|\forall x \varphi(x)\|=\bigwedge_{n \in \omega}\|\varphi(n)\|$

7. $\|\exists \alpha \varphi(\alpha)\|=\bigvee_{\xi \in \Xi}\|\varphi(\xi)\|$

8. $\|\forall \alpha \varphi(\alpha)\|=\bigwedge_{\xi \in \Xi}\|\varphi(\xi)\|$

A sentence $\psi$ is true in the model just in case $\|\psi\|=\top$.

In [5, pages 134-135] Vesley considers a species $R$ of real-number generators: $\xi \in R$ if and only if the sequence $2^{-x} \xi(x)(x \in \omega)$ of diadic fractions is a Cauchy-sequence with $\forall k \exists x \forall p\left|2^{-x} \xi(x)-2^{-x-p} \xi(x+p)\right|<2^{-k}$, i.e. if and only if $\forall k \exists x \forall p 2^{k} \mid 2^{p} \xi(x)-$ $\xi(x+p) \mid<2^{x+p}$.

Equality, ordering, addition and multiplication on $R$ are defined as follows.

1. $\xi=\eta$ if and only if $\forall k \exists x \forall p 2^{k}|\xi(x+p)-\eta(x+p)|<2^{x+p}$,

2. $\xi<\eta$ if and only if $\exists k \exists x \forall p 2^{k}(\eta(x+p) \dot{-} \xi(x+p)) \geq 2^{x+p}$,

3. $(\xi+\eta)(x):=\xi(x)+\eta(x)$ and

4. $(\xi \eta)(x):=\left\lfloor 2^{-x} \xi(x) \eta(x)\right\rfloor$.

The following facts are also proved in [5]. If $\xi, \eta \in R$ then $\xi+\eta \in R,=$ is a congruence relation with respect to $<$ and + . Similar facts are true for multiplication as well (cf. also [6, pages 20-21]).

Let us expand the language $L_{1}$ with a unary predicate symbol $R$, binary predicate symbols $=$ and $<$, and binary function symbols for addition and multiplication on choice sequences.

The corresponding truth values may be defined as follows:

$$
\|R(\xi)\|:=\left\|\forall k \exists x \forall p 2^{k}\left|2^{p} \xi(x)-\xi(x+p)\right|<2^{x+p}\right\|
$$

using obvious abbreviations.

Similarly:

$$
\|\xi=\eta\|:=\left\|\forall k \exists x \forall p 2^{k}|\xi(x+p)-\eta(x+p)|<2^{x+p}\right\|,
$$

and

$$
\|\xi<\eta\|:=\left\|\exists k \exists x \forall p 2^{k}(\eta(x+p) \dot{-} \xi(x+p)) \geq 2^{x+p}\right\| .
$$


Using the facts mentioned above, these definitions can be extended readily to polynomials of choice sequences.

$\xi$ is a global real-number generator just in case $\|R(\xi)\|=\top$. Let $\mathcal{G}$ denote their set. $\mathcal{G}$ is closed under addition and multiplication. From now on elements of $\mathcal{G}$ will be denoted by $f, g, h$ etc.

For each natural number $n$ there is a corresponding global real-number generator $f_{n}$ defined as follows: $f_{n}(l, m)=\top$ if $m=n 2^{l}$ and $f_{n}(l, m)=\perp$ otherwise. Then

$$
\|f_{n} f=\overbrace{f+\cdots+f}^{n}\|=\top .
$$

If it does not cause any confusion, we shall denote $f_{n} \in \mathcal{G}$ by $n$.

Then $\mathcal{G}$ is a model for the language of ordered rings with addition and multiplication defined above where the interpretation of 0 and 1 is $f_{0}$ and $f_{1}$ respectively. Note that $=$ has the usual properties in the model, e.g. $f=g \leftrightarrow \neg(g<f \vee f<g)$, $f=g \wedge \varphi(f) \rightarrow \varphi(g)$ etc. has truth value $\top . \quad f \neq g$ is defined as $f<g \vee g<f$. Finally note that in general $\neg f=g \nLeftarrow \rightarrow f \neq g$.

In what follows a base of a Heyting algebra is a set of elements with the property that every element of the algebra is the supremum of base elements.

If $\mathcal{H}$ has a base of complemented elements, than it is easy to see that quantification over reals in the corresponding model can be reduced to quantification over global real-number generators. I.e. in this case

$$
\|\exists \alpha(R(\alpha) \wedge \varphi(\alpha))\|=\bigvee_{f \in \mathcal{G}}\|\varphi(f)\| \text { and }\|\forall \alpha(R(\alpha) \rightarrow \varphi(\alpha))\|=\bigwedge_{f \in \mathcal{G}}\|\varphi(f)\| .
$$

Definition 1. A Heyting algebra $\mathcal{H}$ is nice if for any $g \in \mathcal{G}$ there is a subalgebra $\mathcal{H}_{1} \leq \mathcal{H}$ containing the elements $g(l, m)$ with the following properties.

1. $\mathcal{H}_{1}$ has a base $\mathcal{D}$ of complemented elements forming a tree of height $\mu$. We shall call $\mu$ the height of the algebra. Each base element $D \in \mathcal{D}$ has a level, an ordinal $\kappa<\mu$ such that $D$ has level $\kappa\left(D \in \mathcal{D}_{\kappa}\right)$ if and only if the following conditions hold.

(i) $D \notin \bigcup_{\lambda<\kappa} \mathcal{D}_{\lambda}$

(ii) $\forall \lambda<\kappa \exists$ ! $D_{\lambda} \in \mathcal{D}_{\lambda}$ such that $D<D_{\lambda}$

(iii) $\forall D^{\prime} \in \mathcal{D}\left(D^{\prime} \in \bigcup_{\lambda<\kappa} \mathcal{D}_{\lambda}\right.$ or $D^{\prime} \wedge D=\perp$ or $\left.D^{\prime} \leq D\right)$

2. If an element of $\mathcal{H}_{1}$ is the supremum of countably many disjoint elements, then it is the supremum of countably many pairwise disjoint complemented elements. 
3. An element $A \in \mathcal{H}_{1}$ is maximal if $A \neq \top$ and for any $B \in \mathcal{H}_{1}$ if $A \leq B \leq \top$, then $B=A$ or $B=\top$. Each $C \in \mathcal{H}_{1}, C \neq \top$ is contained in a maximal element.

In the next lemma we collect some simple facts about $\mathcal{D}$.

Lemma 2. 1. Elements on the same level are pairwise disjoint.

2. Let $D_{i} \in \mathcal{D}_{\lambda_{i}}(i=1,2)$. If $\lambda_{1}<\lambda_{2}$ and $D_{1} \wedge D_{2} \neq \perp$, then $D_{2}<D_{1}$.

3. Let $D \in \mathcal{D}_{\nu}, \mathcal{D}^{\prime}=\left\{D^{\prime} \in \mathcal{D}_{\nu+1} \mid D^{\prime}<D\right\}$. If $\mathcal{D}^{\prime} \neq \emptyset$, let $D_{1}=\bigvee \mathcal{D}^{\prime}$. Then $D=D_{1}$.

4. If $D_{1} \in \mathcal{D}_{\nu}$ and $D_{1}<D_{2}$ then there is some $\lambda<\nu$ such that $D_{2} \in \mathcal{D}_{\lambda}$.

5. For every $n \in \omega$ there is a finest pairwise disjoint cover $\mathcal{C}_{n}$ (ie. $\dot{\vee} \mathcal{C}_{n}=\top$ ) from base elements such that $\mathcal{D}_{n} \subseteq \mathcal{C}_{n}$ and $\mathcal{C}_{n} \subseteq \bigcup\left\{\mathcal{D}_{k} \mid k \leq n\right\}$.

Proof. 1. Immediate from (iii).

2. By (ii) there is a unique $D_{1}^{\prime} \in \mathcal{D}_{\lambda_{1}}$ such that $D_{2}<D_{1}^{\prime}$, so if $D_{1} \wedge D_{2} \neq \perp$, then $D_{1} \wedge D_{1}^{\prime} \neq \perp$. Then from 1 . of this lemma follows that $D_{1}=D_{1}^{\prime}$.

3. We have to show that $D \leq D_{1}$, the other direction is obvious. Assume that $D \not \leq D_{1}$, let $D^{\prime} \in \mathcal{D}^{\prime}$ and $C=D \wedge \neg D^{\prime}$. Then there is a base element $D^{\prime \prime} \leq C$ such that $D " \not \leq D_{1}$. From $D "<D$ and $D " \leq D_{1}$ follows that $D " \in \mathcal{D}_{\lambda}$ for some $\lambda>\nu+1$. By (ii) there is a unique $D_{\nu+1} \in \mathcal{D}_{\nu+1}$ with $D^{\prime \prime}<D_{\nu+1}$. $D \wedge D_{\nu+1} \neq \perp$ follows, so by 2., $D_{\nu+1}<D$ and $D " \leq D_{1}$ follows, a contradiction.

4. Follows from 1. and 2.

5. Follows from (iii) and 3. by induction on $n$. Start with $\mathcal{D}_{\emptyset}$ and refine elements using 3. whenever it is possible.

Lemma 3. 1. Let us assume that $\mathcal{H}$ is a complete Heyting algebra with a base described in the previous definition. Using the notation above assume that $B \neq$ $\top, B \in \mathcal{H}_{1} . B$ contains a set $\mathcal{B}=\left\{B_{i}: i \in I\right\}$ of pairwise disjoint complemented elements of $\mathcal{H}_{1}$ (base elements) such that $B=\bigvee \mathcal{B}$ and for every $C \in \mathcal{H}_{1}$, if $C \vee B>B$ then there is some $i \in I$ with $B_{i} \leq C$.

2. For every element $C$ of $\mathcal{H}_{1}$ there is a countably infinite set of pairwise disjoint elements $\mathcal{U}=\left\{U_{n} \mid n \in \mathbb{N}^{+}\right\}$such that $C=\bigvee \mathcal{U}$. 
3. If the algebra has height $\omega$, then requirement 2 in the definition above follows from 1. in the following stronger form: any element $C$ of $\mathcal{H}_{1}$ is the supremum of countably many pairwise disjoint complemented elements.

Proof. 1. For $\lambda<\mu$ ( $\mu$ is the height of the tree as above) let $\mathcal{B}_{\lambda}=\left\{D \in \mathcal{D}_{\lambda}\right.$ : $D \leq B$ and $\left.\forall \nu<\lambda, \forall E \in \mathcal{B}_{\nu}, E \wedge D=\perp\right\}$.

Let $\mathcal{B}=\bigcup_{\lambda<\mu} \mathcal{B}_{\lambda}$. By definition $\bigvee \mathcal{B} \leq B$. For the other direction assume that there is some base element $D \in \mathcal{D}_{\lambda}$ (here we are using property $(h)$ ) such that $D \notin \bigvee \mathcal{B}$. Then there are $\nu<\lambda$ and $E \in \mathcal{B}_{\nu}$ such that $D \wedge E \neq \perp$. Since $D \in \mathcal{D}_{\lambda}, \nu<\lambda$ and $E \in \mathcal{D}_{\nu}, D<E$. But then $D<\bigvee \mathcal{B}$. So $B=\bigvee \mathcal{B}$.

Now let $C \in \mathcal{H}_{1}, C \vee B>B$. Again there is some $D \in \bigcup_{\lambda} \mathcal{D}_{\lambda}$ such that $D \leq C$ but $D \not \leq B$. Then $D \neq \perp$ and since $\neg B=\perp$ ( $B$ is dense $), D \wedge B \neq \perp$. So there is some $D^{\prime} \in \mathcal{D}_{j}$ with $j<\kappa$ minimal such that $D^{\prime} \leq D \wedge B$.

We claim that $D^{\prime} \in \mathcal{B}_{j}$. Assume that for some $\nu<j$ and $E \in \mathcal{B}_{\nu} E$ and $D^{\prime}$ are not disjoint. Then by properties $(a)$ and $(f)$ above $D^{\prime} \leq E$. Then $D^{\prime} \leq E \wedge D$, so $E \wedge D \neq \perp$. $E \leq D$ would contradict the minimality of $j$, so by property (a) $D<E$, but then $E \not \leq B$ follows, a contradiction again. So $D^{\prime} \in \mathcal{B}_{j}$ and $D^{\prime} \leq C$ proving the claim.

2. Use the construction from 1. inside $C$ and partition the set $\mathcal{B}$ obtained into countably many disjoint sets $\mathcal{B}=\bigcup\left\{\mathcal{B}_{n} \mid n \in \mathbb{N}\right\}$. Let $U_{n}=\bigvee \mathcal{B}_{n}$. Then the set $\mathcal{U}=\left\{U_{n} \mid n \in \mathbb{N}^{+}\right\}$has the required property. Note that since $\mathcal{B}$ is a set of pairwise disjoint elements, $\mathcal{U}$ is a set of pairwise disjoint, but not necessarily complemented elements.

3. Here we use the results and notations of Lemma 2. Since $\mathcal{C}_{n}$ is a disjoint cover for every $n \in \omega$, the supremum $\dot{\bigvee} \mathcal{C}_{n}^{\prime}$ of any subset $\mathcal{C}_{n}^{\prime} \subseteq \mathcal{C}_{n}$ is complemented, if $\mathcal{C}_{n} "=\mathcal{C}_{n} \backslash \mathcal{C}_{n}^{\prime}$ then $\dot{\bigvee} \mathcal{C}_{n}^{\prime} \dot{\vee} \dot{\bigvee} \mathcal{C}_{n} "=\top$ and $\dot{\bigvee} \mathcal{C}_{n}^{\prime} \wedge \dot{\vee} \mathcal{C}_{n} "=\perp$. For $i \in \omega$ let $\mathcal{E}_{i}=\left\{E \in \mathcal{C}_{i} \mid E \leq C \wedge \neg \dot{\bigvee}\left\{E_{j} \mid j<i\right\}\right.$ and $E_{i}=\dot{\bigvee} \mathcal{E}_{i}$. Then, as a supremum of a subset of $\mathcal{C}_{i}, E_{i}$ is complemented. $\mathcal{E}=\left\{E_{i} \mid i \in \omega\right\}$ is a countable set of pairwise disjoint complemented elements. We claim that $C=\dot{\bigvee}\left\{E_{i} \mid i \in \omega\right\} . \dot{\bigvee}\left\{E_{i} \mid i \in \omega\right\} \leq C$ is true by definition. For the other direction, if $C \not \dot{\bigvee}\left\{E_{i} \mid i \in \omega\right\}$ then there is a base element $D_{k} \in \mathcal{D}_{k}$ such that $D_{k} \leq C$ but $D_{k} \not \grave{\bigvee}\left\{E_{i} \mid i \in \omega\right\}$, in particular for every $i \in \omega$ and $E \in \mathcal{E}_{i}$, $D_{k} \not \leq E$. If $i<k$ then, since $\mathcal{C}_{i} \subseteq \bigcup\left\{\mathcal{D}_{j} \mid j \leq i\right\}, E \not \leq D_{k}$. Thus, for every $i<k$ and $E \in \mathcal{E}_{i}, D_{k} \wedge E=\perp$, so $D_{k} \leq C \wedge \neg \dot{\bigvee}\left\{E_{i} \mid i<k\right\}$. But then, since $\mathcal{D}_{k} \subseteq \mathcal{C}_{k}, D_{k} \in \mathcal{E}_{k}$, so $D_{k} \leq \dot{\bigvee}\left\{E_{i} \mid i \in \omega\right\}$, a contradiction. 
Example 1. Let $\kappa$ be any cardinal with the discrete topology, and $\mathcal{H}$ be the Heyting algebra of the open sets of $X={ }^{\omega} \kappa$. Then $\mathcal{H}$ with $\mathcal{H}_{1}=\mathcal{H}$ fulfills the requirements of Definition 1. Note that the open set algebra gives us Scott's model in the case of $\kappa=\omega($ see $[3])$.

Proof. The height of the tree of base elements required in Definition1.1. is $\omega$. For $\sigma \in$ ${ }^{n} \kappa(n \in \omega)$ let $B_{\sigma}=\left\{p \in{ }^{\omega} \kappa \mid \sigma \subset p\right\}$. Let $\mathcal{D}_{n}=\left\{B_{\sigma} \mid \sigma \in{ }^{n} \kappa\right\}$ and $\mathcal{D}=\bigcup_{n \in \omega} \mathcal{D}_{n}$. For every $p \in{ }^{\omega} \kappa, U_{p}={ }^{\omega} \kappa \backslash\{p\}$ is a maximal element and $\bigwedge\left\{U_{p} \mid p \in{ }^{\omega} \kappa\right\}=\perp$.

Example 2. Let again $\kappa$ be an infinite cardinal, but now let $\mathcal{H}$ be the Heyting algebra of the open sets of $X={ }^{\kappa}\left({ }^{\omega} 2\right)$ with the product topology. For each $g \in \mathcal{G}$ there is a subalgebra $\mathcal{H}_{1}$ isomorphic to the open-set algebra of ${ }^{\omega} 2$ and containing the elements $g(m, n)$ - the subalgebra of elements with support equal to the support of $g$ (see [7]). Then $\mathcal{H}$ is nice, this can be shown by using the isomorphism between $\mathcal{H}_{1}$ and the open-set algebra of ${ }^{\omega_{2}} 2$ and the previous example. Note that if $\kappa>\omega$ than these models are elementarily equivalent to Krol's model defined in [8] (cf. [7]).

Note. The results in [1] were true for a third class of Heyting algebras, the algebras of the coperfect open sets of $X={ }^{\omega} \kappa$. In particular true first order arithmetic can be interpreted in the corresponding real algebras (see below). These Heyting algebras however have no maximal elements, so the proof of the interpretability of second order arithmetic below does not go through.

Definition 4. Let $h_{1}, h_{2} \in \mathcal{G}$ global real number generators, $B \in \mathcal{H}_{1}$. A positive natural number $n$ is an NE-quotient (non-excluded quotient) of $h_{1}, h_{2}$ with respect to $B$ if $\left\|\neg n h_{2}^{W}=h_{1}\right\| \leq B$.

We shall encode subsets of natural numbers as NE-quotients of appropriate elements of $\mathcal{G}$.

Definition 5. Using the notation above, for each $W \subseteq \mathbb{N}$ we define the encoding real number generators in a nice Heyting algebra $\mathcal{H}$ as follows. Let us assume that $B \neq \top, \mathcal{B}=\left\{B_{i}: i \in I\right\}$ has the property of the previous lemma. For every $i \in I$ let $\left\{U_{i}^{n} \neq \perp: n \in \mathbb{N}^{+}\right\}$be a set of disjoint elements with $\bigvee_{n \in \mathbb{N}^{+}} U_{i}^{n}=B_{i}$ (using property 2. of nice Heyting algebras) and $U_{i}^{1}=B_{i} \wedge \neg \bigvee_{n>1} U_{i}^{n}$. Let $U^{n}=\bigvee_{i \in I} U_{i}^{n}$. Using property 3., let $\bigvee_{n \in \mathbb{N}^{+}} U^{n}=\bigvee_{i \in \omega} E_{i}$ where $\left\{E_{i}\right\}$ is a countable sequence of pairwise disjoint complemented elements. Let $W \subseteq \mathbb{N}^{+}, W \neq \emptyset, F: \mathbb{N}^{+} \rightarrow W$ an onto function. Let

$$
h_{1}(l, m)= \begin{cases}\left(\bigvee_{i \leq l} E_{i}\right)^{c} & \text { if } m=0 \\ E_{i} & \text { if } m=2^{l-i},(0 \leq i \leq l) \\ \perp & \text { otherwise, }\end{cases}
$$




$$
h_{2}^{W}(l, m)= \begin{cases}\left(\bigvee_{i \leq l} E_{i}\right)^{c} & \text { if } m=0 \\ \bigvee\left\{E_{i} \wedge U^{n}: m=\left\lceil\frac{2^{l-i}}{F(n)}\right\rceil, 0 \leq i \leq l\right\} & \text { if } m=\left\lceil\frac{2^{l-i}}{F(n)}\right\rceil \text { for some } n \text { and } i \\ \perp & \text { otherwise. }\end{cases}
$$

Note that $m=\left\lceil\frac{k}{n}\right\rceil$ ( $m$ is the least integer greater than or equal to the rational number $k / n)$ is definable in our language as $k \leq m n \wedge m n<k+n$.

If $W=\mathbb{N}^{+}$then we shall use the notation $h_{2}$ for $h_{2}^{\mathbb{N}}$ with the function $F$ being the identity function.

For $W=\emptyset$ let $h_{2}^{\emptyset}=0$.

Note that every element of the form $h_{2}^{W}(m, k)$ and $h_{1}(m, k)$ is in $\mathcal{H}_{1}$.

Lemma 6. Using the notation above here we extend and modify Lemma 3. in [1].

1. $U^{l} \wedge U^{m}=\perp$ if $l \neq m$.

2. $\neg \bigvee_{n \in \mathbb{N}^{+}} U^{n}=\neg B=\perp$.

3. $h_{2}^{W}, h_{1} \in \mathcal{G}$, ie. $\left\|R\left(h_{2}^{W}\right)\right\|=\left\|R\left(h_{1}\right)\right\|=\top$.

4. $\bigvee_{n \in \mathbb{N}^{+}} U^{n}=\left\|h_{1} \neq 0\right\|=\left\|h_{2}^{W} \neq 0\right\|$

5. $U^{n} \leq\left\|F(n) h_{2}^{W}=h_{1}\right\|$

6. $\neg \bigvee_{n \in \mathbb{N}^{+}}\left\|F(n) h_{2}^{W}=h_{1}\right\|=\left\|h_{1}=0\right\|=\left\|h_{2}^{W}=0\right\|=\neg \bigvee_{n \in \mathbb{N}^{+}} U^{n}=\perp$

7. For all $k \in \mathbb{N}^{+},\left\|\neg k h_{2}^{W}=h_{1}\right\| \leq B$ if and only if $k=F(n)$ for some $n \in \mathbb{N}$, ie. $W$ is the set of $N E$-quotients of $h_{1}, h_{2}^{W}$ with respect to $B$. In particular for all $n \in \mathbb{N}^{+},\left\|\neg n h_{2}=h_{1}\right\| \leq B$.

8. For all $k, l \in \mathbb{N}^{+}$if $k \neq l$ then $\left\|l h_{2}^{W}=h_{1}\right\| \leq\left\|\neg k h_{2}^{W}=h_{1}\right\|$.

9. For all $n \in \mathbb{N}^{+}\left\|n h_{2}=h_{1}\right\| \leq\left\|\neg \neg F(n) h_{2}^{W}=h_{1}\right\|$.

10. For all $n, k \in \mathbb{N}^{+}$if $k \neq F(n)$ then $\left\|n h_{2}=h_{1}\right\| \leq\left\|\neg k h_{2}^{W}=h_{1}\right\|$.

11. $\neg \neg \bigvee_{n \in \mathbb{N}^{+}}\left\|F(n) h_{2}^{W}=h_{1}\right\|=\top$.

12. If for all $n \in \mathbb{N}^{+}$if $k \neq F(n)$ then $\left\|\neg k h_{2}^{W}=h_{1}\right\|=\top$.

13. If $W=\emptyset,\left\|\neg k h_{2}^{\emptyset}=h_{1}\right\|=\top$ for every $k \in \mathbb{N}^{+}$.

14. For any element $B \in \mathcal{H}_{1}$ there is $g \in \mathcal{G}$ such that $B=\|g \neq 0\|$

Proof. First assume that $W \neq \emptyset$. 
1. $U^{l} \wedge U^{m}=\perp$ if $l \neq m$ immediately follows from the definition.

2. $\neg B=\perp$ by assumption ( $B$ is dense). For $\neg \bigvee_{n \in \mathbb{N}^{+}} U^{n}=\perp$ we have

$$
\begin{aligned}
& \neg \bigvee_{n \in \mathbb{N}^{+}} U^{n}=\neg \bigvee_{n \in \mathbb{N}^{+}} \bigvee_{i \in I} U_{i}^{n}=\bigwedge_{i \in I} \bigwedge_{n \in \mathbb{N}^{+}} \neg U_{i}^{n}=\bigwedge_{i \in I}\left(\neg U_{i}^{1} \wedge \bigwedge_{n>1} \neg U_{i}^{n}\right) \\
= & \bigwedge_{i \in I}\left(\neg\left(B_{i} \wedge \neg \bigvee_{n>1} U_{i}^{n}\right) \wedge \neg \bigvee_{n>1} U_{i}^{n}\right) \leq \bigwedge_{i \in I} \neg B_{i}=\neg \bigvee_{i \in I} B_{i}=\neg B=\perp
\end{aligned}
$$

3. $h_{2}^{W} \in \mathcal{G}$, ie. $\left\|R\left(h_{2}^{W}\right)\right\|=\top$. $\left(\left\|R\left(h_{1}\right)\right\|=\top\right.$ is similar. $)$

First of all $h_{2}^{W}$ is a choice sequence, since $h_{2}^{W}(l, m)$ and $h_{2}^{W}(l, n)$ are obviously disjoint if $m \neq n$, and

$$
\begin{gathered}
\bigvee_{m \in \omega} h_{2}^{W}(l, m)=\left(\bigvee_{i \leq l} E_{i}\right)^{c} \vee \bigvee_{i \leq l} \bigvee_{n \in \mathbb{N}^{+}}\left(E_{i} \wedge U^{n}\right)= \\
\left(\bigvee_{i \leq l} E_{i}\right)^{c} \vee \bigvee_{i \leq l}\left(E_{i} \wedge\left(\bigvee_{n \in \mathbb{N}^{+}} U^{n}\right)\right)=\left(\bigvee_{i \leq l} E_{i}\right)^{c} \vee \bigvee_{i \leq l}\left(E_{i} \wedge\left(\bigvee_{j \in \omega} E_{j}\right)\right)=\top .
\end{gathered}
$$

Next we want to show that $\top \leq\left\|\forall k \exists x \forall p 2^{k}\left|2^{p} h_{2}^{W}(x)-h_{2}^{W}(x+p)\right|<2^{x+p}\right\|$. Fix $k \in \omega$ and let $x>k$. Then for all $i \leq x, p \in \omega$ and $n \in \mathbb{N}^{+}$,

$$
E_{i} \wedge U^{n} \leq\left\|h_{2}^{W}(x+p)=\left\lceil\frac{2^{x+p-i}}{F(n)}\right\rceil\right\|
$$

Then

$$
E_{i} \wedge U^{n} \leq\left\|2^{x+p-i} \leq h_{2}^{W}(x+p) F(n) \wedge h_{2}^{W}(x+p) F(n) \leq 2^{x+p-i}+F(n)\right\|
$$

and

$$
E_{i} \wedge U^{n} \leq\left\|2^{x+p-i} \leq 2^{p} h_{2}^{W}(x) F(n) \wedge 2^{p} h_{2}^{W}(x) F(n) \leq 2^{x+p-i}+2^{p} F(n)\right\|
$$

we have

$$
E_{i} \wedge U^{n} \leq\left\|2^{k}\left|2^{p} h_{2}^{W}(x)-h_{2}^{W}(x+p)\right|<2^{k}\left(2^{p}+1\right)\right\| \wedge\left\|2^{k}\left(2^{p}+1\right) \leq 2^{x+p}\right\| .
$$

Also,

$$
\left(\bigvee_{i \leq x} E_{i}\right)^{c} \leq\left\|h_{2}^{W}(x)=0\right\| \wedge\left\|h_{2}^{W}(x+p) \leq 2^{x+p-(x+1)}\right\| \wedge\left\|2^{x+p-(x+1)}=2^{p-1}\right\|
$$


for all $p$, so

$$
\left(\bigvee_{i \leq x} E_{i}\right)^{c} \leq \bigwedge_{p \in \omega}\left\|2^{k}\left|2^{p} h_{2}^{W}(x)-h_{2}^{W}(x+p)\right| \leq 2^{k+p-1}\right\| \wedge\left\|2^{k+p-1}<2^{x+p}\right\| .
$$

From these

$$
\top \leq\left\|\forall k \exists x \forall p 2^{k}\left|2^{p} h_{2}^{W}(x)-h_{2}^{W}(x+p)\right|<2^{x+p}\right\|
$$

follows.

4. $\bigvee_{n \in \mathbb{N}^{+}} U^{n}=\left\|h_{1} \neq 0\right\|=\left\|h_{2}^{W} \neq 0\right\|$.

We show only that $\bigvee_{n \in \mathbb{N}^{+}} U^{n}=\left\|h_{2}^{W} \neq 0\right\|, \bigvee_{n \in \mathbb{N}^{+}} U^{n}=\left\|h_{1} \neq 0\right\|$ is similar.

Since $\left(\bigvee_{i \leq x+p} E_{i}\right)^{c} \leq\left\|h_{2}^{W}(x+p)=0\right\|,\left\|2^{k} h_{2}^{W}(x+p) \geq 2^{x+p}\right\| \leq \bigvee_{i \leq x+p} E_{i}$

From this follows that

$$
\begin{aligned}
\left\|h_{2}^{W} \neq 0\right\|= & \bigvee \bigvee_{k \in \omega} \bigwedge_{x \in \omega}\left\|2^{k} h_{2}^{W}(x+p) \geq 2^{x+p}\right\| \leq \bigvee_{k \in \omega} \bigvee_{x \in \omega} \bigwedge_{p \in \omega} \bigvee_{i \leq x+p} E_{i}= \\
& \bigvee \bigvee_{k \in \omega} \bigvee_{x \in \omega} E_{i}=\bigvee \bigvee_{k \in \omega} \bigvee_{x \in \omega} E_{x}=\bigvee_{x \in \omega} E_{x}=\bigvee_{n \in \mathbb{N}^{+}} U^{n} .
\end{aligned}
$$

On the other hand for $n \in \mathbb{N}^{+}$and $i \in \omega$ fixed, if $F(n)<2^{y}$ and $x=k=y+i$, then for all $p \in \omega$,

$$
\begin{gathered}
E_{i} \wedge U^{n} \leq\left\|2^{k} h_{2}^{W}(x+p)=2^{k}\left\lceil\frac{2^{x+p-i}}{F(n)}\right\rceil\right\| \wedge\left\|2^{k}\left\lceil\frac{2^{x+p-i}}{F(n)}\right\rceil>2^{k+x+p-i-y}\right\| \wedge \\
\left\|2^{k+x+p-i-y}=2^{x+p}\right\| .
\end{gathered}
$$

From this $\bigvee_{n \in \mathbb{N}^{+}} U^{n} \leq\left\|h_{2}^{W} \neq 0\right\|$ follows.

5. We claim that for all $n \in \mathbb{N}^{+}$and $i \in \omega, E_{i} \wedge U^{n} \leq\left\|F(n) h_{2}^{W}=h_{1}\right\|$. Since $U^{n}=\bigvee_{i \in \omega}\left(E_{i} \wedge U^{n}\right)$, from this follows that $U^{n} \leq\left\|F(n) h_{2}^{W}=h_{1}\right\|$ as claimed. If $i \leq l$ then

$$
E_{i} \wedge U^{n} \leq\left\|h_{2}^{W}(l)=\left\lceil\frac{2^{l-i}}{F(n)}\right\rceil\right\| \wedge\left\|h_{1}(l)=2^{l-i}\right\|,
$$

so for all $k \in \omega$, if $x>k, x>i$ and $2^{x}>F(n) 2^{k}$, then for all $p \in \omega$,

$$
E_{i} \wedge U^{n} \leq\left\|2^{k}\left|F(n) h_{2}^{W}(x+p)-h_{1}(x+p)\right|<F(n) 2^{k}\right\| \wedge\left\|F(n) 2^{k}<2^{x+p}\right\| .
$$

From this the statement follows. 
6. $\neg \bigvee_{n \in \mathbb{N}^{+}}\left\|F(n) h_{2}^{W}=h_{1}\right\|=\left\|h_{1}=0\right\|=\left\|h_{2}^{W}=0\right\|=\neg \bigvee_{n \in \mathbb{N}^{+}} U^{n}=\perp$ follows from 2., 4. and 5.

7. First we show that for all $n \in \mathbb{N}^{+}\left\|\neg F(n) h_{2}^{W}=h_{1}\right\| \leq B$. Otherwise $\| \neg F(n) h_{2}^{W}=$ $h_{1} \| \vee B>B$, so by Lemma 3. $B_{i} \leq\left\|\neg F(n) h_{2}^{W}=h_{1}\right\|$ for some $i \in I$. Thus $U_{i}^{n} \leq\left\|\neg F(n) h_{2}^{W}=h_{1}\right\|$, but $U_{i}^{n} \leq\left\|F(n) h_{2}^{W}=h_{1}\right\|$, a contradiction.

Now let us assume that $k \notin W$. Then $\left\|k h_{2}^{W}=h_{1}\right\| \wedge \bigvee_{n \in \mathbb{N}}\left\|F(n) h_{2}^{W}=h_{1}\right\|=\perp$, so $\left\|k h_{2}^{W}=h_{1}\right\| \leq \neg \bigvee_{n \in \mathbb{N}}\left\|F(n) h_{2}^{W}=h_{1}\right\|=\perp$ and $\left\|\neg k h_{2}^{W}=h_{1}\right\|=\top$ and thus $k$ is not an NE-quotient.

8. Assume that $k \neq l \in \mathbb{N}^{+}$. Then $\left\|l h_{2}^{W}=h_{1}\right\| \wedge\left\|k h_{2}^{W}=h_{1}\right\|=\left\|l h_{2}^{W}=h_{1}\right\| \wedge$ $\left\|k h_{2}^{W}=h_{1}\right\| \wedge\|k \neq l\| \leq\left\|h_{2}^{W}=0\right\|=\perp$, so $\left\|l h_{2}^{W}=h_{1}\right\| \leq\left\|\neg k h_{2}^{W}=h_{1}\right\|$.

9. For $n \in \mathbb{N}^{+}$let $A_{n}=\left\|n h_{2}=h_{1}\right\| \wedge\left\|\neg F(n) h_{2}^{W}=h_{1}\right\|$. We claim that $A_{n}=\perp$, from this $\left\|n h_{2}=h_{1}\right\| \leq\left\|\neg \neg F(n) h_{2}^{W}=h_{1}\right\|$ follows. Since $A_{n} \leq\left\|n h_{2}=h_{1}\right\|$ they are pairwise disjoint. Also, for all $n \in \mathbb{N}^{+} A_{n} \wedge U^{n}=\perp$, since $A_{n} \leq$ $\left\|\neg F(n) h_{2}^{W}=h_{1}\right\|$ and $U^{n} \leq\left\|F(n) h_{2}^{W}=h_{1}\right\|$. From 3. and the fact that $U^{k} \leq\left\|k h_{2}=h_{1}\right\|$ and $A_{n} \leq\left\|n h_{2}=h_{1}\right\|$ follows that if $k \neq n$ then $U^{k} \wedge A_{n}=\perp$. So $A_{n} \vee\left(\bigvee_{k \in \mathbb{N}^{+}} U^{k}\right)$ is a disjoint union. Then from $\neg \bigvee_{n \in \mathbb{N}^{+}} U^{n}=\perp$ (see 1.) follows that $A_{n}=\perp$ as claimed.

10. Assume $k \neq F(n)$. From 8. $\left\|F(n) h_{2}^{W}=h_{1}\right\| \leq\left\|\neg k h_{2}^{W}=h_{1}\right\|$, so $\| \neg \neg F(n) h_{2}^{W}=$ $h_{1}\|\leq\| \neg \neg \neg k h_{2}^{W}=h_{1} \|$, ie. $\left\|\neg \neg F(n) h_{2}^{W}=h_{1}\right\| \leq\left\|\neg k h_{2}^{W}=h_{1}\right\|$. By 9 . $\left\|n h_{2}=h_{1}\right\| \leq\left\|\neg \neg F(n) h_{2}^{W}=h_{1}\right\|$. From these $\left\|n h_{2}=h_{1}\right\| \leq\left\|\neg k h_{2}^{W}=h_{1}\right\|$ as claimed.

11. $\neg \neg \bigvee_{n \in \mathbb{N}^{+}}\left\|F(n) h_{2}^{W}=h_{1}\right\|=\top$ follows from $\neg \bigvee_{n \in \mathbb{N}^{+}}\left\|F(n) h_{2}^{W}=h_{1}\right\|=\perp$

12. If for all $n \in \mathbb{N}^{+}$if $k \neq F(n)$ then by 10. for all $n \in \mathbb{N}^{+}\left\|n h_{2}=h_{1}\right\| \leq$ $\left\|\neg k h_{2}^{W}=h_{1}\right\|$, ie. $\bigvee_{n \in \mathbb{N}^{+}}\left\|n h_{2}=h_{1}\right\| \leq\left\|\neg k h_{2}^{W}=h_{1}\right\|$. Then by 11 . $\top=$ $\neg \neg \bigvee_{n \in \mathbb{N}^{+}}\left\|n h_{2}=h_{1}\right\| \leq \neg \neg\left\|\neg k h_{2}^{W}=h_{1}\right\|=\left\|\neg k h_{2}^{W}=h_{1}\right\|$ and the statement follows.

13. $\left\|h_{2}^{\emptyset}=0\right\|=\top$ by definition, so $\left\|k h_{2}^{\emptyset}=h_{1}\right\| \leq\left\|h_{1}=0\right\|=\perp$ and $\| \neg k h_{2}^{\emptyset}=$ $h_{1} \|=\top$.

14. Use the construction of the previous definition. 


\section{Coding $\mathbb{N}^{+}$}

This section is from [1]. The variables $x, y, u, v, w$ etc. range over reals, $k, l, n$ will range over natural numbers. From now on let $B(y)$ denote the L-formula $y=0 \vee y \neq 0$. Let $\varphi_{\mathbb{N}^{+}}(x, y, u, v) \equiv$

$$
\begin{gathered}
\neg x<1 \wedge(\neg v=u \vee \neg x v=u \rightarrow B(y)) \wedge \forall w[(\neg w v=u \rightarrow B(y)) \rightarrow \\
\left.\left[(w<1 \rightarrow B(y)) \wedge\left(w>1 \rightarrow \exists w^{\prime}\left(w \neq w^{\prime} \vee \neg w^{\prime} v=u+v \rightarrow B(y)\right)\right)\right]\right] .
\end{gathered}
$$

Note that $x \neq y$ is defined as $x<y \vee y<x$ and $\neg x=y$, which is equivalent to $\neg \neg x \neq y$, is weaker intuitionistically than $x \neq y$.

The following sentences are used as axioms, they are true in the models of intuitionistic second-order arithmetic we have mentioned:

1. $\forall y \exists u \exists v\left(\forall n \in \mathbb{N}^{+}\left(\neg n v=u \rightarrow B(y) \wedge \neg v=0 \wedge \neg \neg \exists n \in \mathbb{N}^{+}(n v=u)\right)\right.$;

2. $\neg \forall y B(y)$.

Note that if $y=0 \vee y \neq 0$ is true, then in 1 . arbitrary $u=v \neq 0$ work, so this sentence is true classically.

Theorem 7. (From [1]) Let $\psi_{\mathbb{N}^{+}}(x)$ denote the L-formula $\forall y \exists u \exists v \varphi_{\mathbb{N}^{+}}(x, y, u, v)$. Then from the axioms and the properties of real numbers mentioned above and from the usual axioms of natural numbers $\exists k \in \mathbb{N}^{+}(x=k) \equiv \psi_{\mathbb{N}^{+}}(x)$ follows in two-sorted intuitionistic predicate calculus with equality. In particular the statement holds in our models: for all $h \in \mathcal{G}, \bigvee_{k \in \mathbb{N}^{+}}\|h=k\|=\left\|\psi_{\mathbb{N}^{+}}(h)\right\|$.

\section{Coding Second-Order Arithmetic}

Let $\varphi(\vec{x}, \vec{S})$ be a second-order formula of the language $L^{\prime}=\langle 1,+, \times\rangle$. Here $\vec{x}$ is a tuple of first-order variables, $\vec{S}$ is a tuple of second-order variables. Without loss of generality, we assume that $\varphi$ does not contain any implications. $\varphi_{1}(S / \emptyset)$ denotes the formula obtained from $\varphi$ by regarding $S$ as the empty set: replace all subformulas of $\varphi$ of the form $x \in S$ where $x$ is a numeric variable with the (false) formula $\neg x=x$. For each second-order variable $S$ let $v_{S}$ be a new variable, and let $y$ be a new variable occuring only in the indicated places. The formula $\varphi$ will be encoded by $\tilde{\varphi}\left(\vec{x}, \vec{v}_{S}, y, u\right)$, a formula of the language of ordered rings. Here the free variables in $\vec{x}$ correspond 
to free natural number variables in $\varphi, \vec{v}_{S}$ corresponds to free second order variables in $\varphi, y$ corresonds to $g \in \mathcal{G}$ above, $u$ to $h_{1}$ and $v$ to $h_{2}$, the pair of reals in the model encoding the set of natural numbers. $\tilde{\varphi}$ is defined inductively as follows $(\tilde{\varphi}$ may contain other variables than the ones indicated):

(i) If $\varphi$ is first-order atomic, then $\tilde{\varphi} \equiv \neg \varphi \rightarrow y \neq 0$;

(ii) $\widetilde{x \in S} \equiv \neg x v_{S}=u \rightarrow y \neq 0$;

(iii) $\widetilde{\varphi_{1} \circ \varphi_{2}} \equiv \tilde{\varphi}_{1} \circ \tilde{\varphi}_{2}$ where $\circ=\wedge, \vee$;

(iv) $\widetilde{\neg \varphi_{1}} \equiv \tilde{\varphi}_{1} \rightarrow y \neq 0$;

(v) $\widetilde{\exists x \varphi_{1}(x)} \equiv \exists x\left(\psi_{\mathbb{N}^{+}}(x) \wedge \tilde{\varphi}_{1}(x)\right)$;

(vi) $\widetilde{\forall x \varphi_{1}(x)} \equiv \forall x\left(\psi_{\mathbb{N}^{+}}(x) \rightarrow \tilde{\varphi}_{1}(x)\right)$;

(vii) $\exists \widetilde{S \varphi_{1}(S)} \equiv \widetilde{\varphi_{1}(S / \emptyset)} \vee \exists v_{S} \exists u \tilde{\varphi}_{1}\left(v_{S}, u\right)$;

(viii) $\forall \widetilde{S \varphi_{1}(S)} \equiv \forall v_{S} \forall u \tilde{\varphi}_{1}\left(v_{S}, u\right)$.

In the next lemmas let $g$ be a fixed element of $\mathcal{G}$ used in the definition of $h_{2}^{W}$ and $h_{1}$, let $A_{g}=\|g \neq 0\|$ and let $C_{g}=\|\zeta(g)\|$. For $k \in \mathbb{N}^{+} k$ also denote the corresponding real-number generator as before.

Definition 8. Let $U \in \mathcal{H}_{1}$. An element $C \in \mathcal{H}_{1}$ is maximal in $U$ if $C<U$ and for any $C^{\prime} \in \mathcal{H}_{1}$ if $C \leq C^{\prime} \leq U$ then either $C^{\prime}=C$ or $C^{\prime}=U$.

Lemma 9. Let $A<\top$ and $M$ maximal. If $A<M \rightarrow A$, then $A$ is maximal in $M \rightarrow A$.

Proof. Let $A \leq C \leq M \rightarrow A$. If $M=M \wedge C$, then $C \leq M$, so since $C \leq M \rightarrow A$, $C \leq A$ and $C=A$ follows. Otherwise $M<M \vee C$. Then, since $M$ is maximal by assumption, $M \vee C=\top$, so $M \rightarrow A=(M \rightarrow A) \wedge(M \vee C)=((M \rightarrow A) \wedge M) \vee((M \rightarrow$ $A) \wedge C) \leq A \vee C=C$, so $C=M \rightarrow A$ and we are done.

Lemma 10. Let $A<\top$. For each complemented $D \not \leq A$ there is an element $B \in \mathcal{H}_{1}$ such that $B \leq D \vee A$ and $A=B$ or $A$ is maximal in $B$. 
Proof. Let $E$ be a maximal element such that $\neg D \vee A \leq E$. By our assumption on $\mathcal{H}_{1}$ such an element exists. If $E=A$, then $B=\top$ fulfills the requirements. If $A$ is maximal in $E$, then with $B=E$ we are done. Otherwise let $B=E \rightarrow A$. First we claim that $E \rightarrow A \leq D \vee A$. $E \rightarrow A \leq(\neg D \vee A) \rightarrow A \leq \neg D \rightarrow A=(\neg D \rightarrow$ $A) \wedge(D \vee \neg D)=((\neg D \rightarrow A) \wedge D) \vee A \leq D \vee A$.

Next assume that $A<B$. Then $A$ is maximal in $B$ by Lemma 9 .

Lemma 11. 1. If $C \in \mathcal{H}_{1}$ is maximal with $C=\|g \neq 0\|$ for some $g \in \mathcal{G}$ in $B$, then $B \leq\|\zeta(g)\|$.

2. Let $A_{g}<C_{g}$ (notation as above) and let $\mathcal{C}=\left\{C \leq C_{g} \mid A_{g}\right.$ is maximal in $\left.C\right\}$. Then $\bigvee \mathcal{C}=C_{g}$.

3. $C=\bigwedge\left\{B \in \mathcal{H}_{1} \mid B\right.$ is maximal $\}=\perp$

Proof. 1. Let $h \in \mathcal{G}$ arbitrary, $A=\|h \neq 0\|$. We have to show that $B \leq(A \rightarrow$ $C) \vee A$. Since $C$ is maximal, either $C=(C \vee A) \wedge B$, or $(C \vee A) \wedge B=B$. In the first case $C=(C \vee A) \wedge B=(C \wedge B) \vee(A \wedge B)=C \vee(A \wedge B)$, so $A \wedge B \leq C$ and then $B \leq A \rightarrow C$, so $B \leq(A \rightarrow C) \vee A$. In the second case $B=(C \vee A) \wedge B=(C \wedge B) \vee(A \wedge B) \leq(A \rightarrow C) \vee A$ and we are done.

2. Assume that $C_{g} \not \mathbb{V} \bigvee$. Then there is a complemented $D \in \mathcal{H}_{1}$ such that $D \leq C_{g}$ and $D \not \leq \vee \mathcal{C}$. By Lemma 10. there is $B \leq D \vee C_{g}$ such that $A_{g}$ is maximal in $B . \neg D \vee \vee \mathcal{C}<\top$, so there is a maximal element $M$ such that $\neg D \vee \vee \mathcal{C} \leq M$. There is $h \in \mathcal{G}$ such that $M=\|h \neq 0\|$. If $A_{g}=M \rightarrow A_{g}$, then $D \leq C_{g} \leq\left(M \rightarrow A_{g}\right) \vee M=A_{g} \vee M$, so $\top=D \vee \neg D \leq A_{g} \vee M$, but $A_{g} \leq \bigvee \mathcal{C} \leq M$, so $M=\top$ follows, a contradiction. So $A_{g}<M \rightarrow A_{g}$ and by Lemma $9 A_{g}$ is maximal in $M \rightarrow A_{g}$. Thus by part $1 . M \rightarrow A_{g} \leq C_{g}$, so $M \rightarrow A_{g} \in \mathcal{C}$ and $M \rightarrow A_{g} \leq \bigvee \mathcal{C} \leq M$. Then $M \rightarrow A_{g} \leq A_{g}$, a contradiction.

3. Suppose that $C>\perp$ and let $D<C$ a complemented element. Then $\neg D$ is contained in a maximal element $M \neq \top$, so $\top=D \vee \neg D \leq C \vee \neg D \leq M$, a contradiction.

Lemma 12. Using the notation above, for each second order $L^{\prime}$-formula $\varphi$, $A_{g} \leq\|\tilde{\varphi}(g)\|$.

Proof. By formula induction.

Lemma 13. Let $\varphi(\vec{x}, \vec{S})$ be a second-order $L^{\prime}$-formula, $\vec{a}$ be a tuple of positive integers, and $\vec{W}$ be a tuple of subsets of $\mathbb{N}^{+}$. Let $B$ be an arbitrary element such that $A_{g}$ is maximal in $B$. Using the notation of Theorem 6 ( $\tilde{\varphi}$ may contain other parameters than the ones indicated): 
1. If $\mathbb{N}^{+}=\varphi[\vec{a}, \vec{W}]$ then $\left\|\tilde{\varphi}\left[\vec{a}, \vec{h}_{2}^{W}, g, h_{1}\right]\right\| \geq B$

2. If $\mathbb{N}^{+} \not=\varphi[\vec{a}, \vec{W}]$ then $\left\|\tilde{\varphi}\left[\vec{a}, \vec{h}_{2}^{W}, g, h_{1}\right]\right\| \leq A_{g}$

3. Let $h_{2}^{\prime}, h_{1}^{\prime}$ be an arbitrary pair of elements of $\mathcal{G}$,

let $W=\left\{k \in \mathbb{N}^{+} \mid\left\|\neg k h_{2}^{\prime}=h_{1}^{\prime}\right\| \rightarrow A_{g} \geq B\right\}, h_{2}^{W}$ the element of $\mathcal{G}$ corresponding to $W$, then if $W \neq \emptyset$,

$$
\begin{aligned}
\left\|\tilde{\varphi}\left[h_{2}^{\prime}, g, h_{1}^{\prime}\right]\right\| \geq B & \Leftrightarrow\left\|\tilde{\varphi}\left[h_{2}^{W}, g, h_{1}\right]\right\| \geq B \\
\left\|\tilde{\varphi}\left[h_{2}^{\prime}, g, h_{1}^{\prime}\right]\right\| \leq A_{g} & \Leftrightarrow\left\|\tilde{\varphi}\left[h_{2}^{W}, g, h_{1}\right]\right\| \leq A_{g}
\end{aligned}
$$

Ie. each element $h_{2}^{\prime}$ of $\mathcal{G} \theta$ can be regarded as $h_{2}^{W}$ for the appropriate subset $W \subseteq \mathbb{N}^{+}$.

Proof. By formula induction.

(i) If $\varphi$ is first-order atomic and $\mathbb{N}^{+} \models \varphi$ then $\|\neg \varphi\|=\perp$ and $\|\neg \varphi\| \rightarrow A_{g}=\top$. If $\mathbb{N}^{+} \forall=\varphi$, then $\|\neg \varphi\|=\top$ and $\|\neg \varphi\| \rightarrow A_{g}=A_{g}$. Finally 3. holds since $\tilde{\varphi}$ does not contain the variable $v_{S}$.

(ii) $\widetilde{x \in S} \equiv \neg x v_{S}=u \rightarrow B(y)$.

1. follows from Theorem 6.7: $\left\|\neg a h_{2}^{W}=h_{1}\right\| \rightarrow A_{g}=\top \geq B$.

2. Assume that $\mathbb{N}^{+} \forall a \in W$. It is enough to show that $\left\|\neg a h_{2}^{W}=h_{1}\right\|=\top$. First assume that $W \neq \emptyset$ and let $F: \mathbb{N}^{+} \rightarrow W$ be the surjection corresponding to $W$. Then $\forall n \in \mathbb{N}^{+} F(n) \neq a$, so by Theorem 6.10. $\forall n \in \mathbb{N}^{+}\left\|n h_{2}=h_{1}\right\| \leq$ $\left\|\neg a h_{2}^{W}=h_{1}\right\|$, so $\bigvee_{n \in \mathbb{N}^{+}}\left\|n h_{2}=h_{1}\right\| \leq\left\|\neg a h_{2}^{W}=h_{1}\right\|$. Using Theorem 6.11, $\top=\neg \neg \bigvee_{n \in \mathbb{N}^{+}}\left\|n h_{2}=h_{1}\right\| \leq \neg \neg\left\|\neg a h_{2}^{W}=h_{1}\right\|=\left\|\neg a h_{2}^{W}=h_{1}\right\|$.

If $W=\emptyset$ then $\left\|\neg a h_{2}^{W}=h_{1}\right\|=\top$ by Theorem 6.13.

3. If $a \in W$ then by 1 . $\left\|\neg a h_{2}^{W}=h_{1}\right\| \rightarrow A_{g} \geq B$. Also, by the definition of $W$, $\left\|\neg a h_{2}^{\prime}=h_{1}^{\prime}\right\| \rightarrow A_{g} \geq B$.

If $a \notin W$ then $\left\|\neg a h_{2}^{W}=h_{1}\right\|=\top$ by Theorem 6.12 , so $\left\|\neg a h_{2}^{W}=h_{1}\right\| \rightarrow A_{g}=$ $A_{g}$. By the definition of $W,\left\|\neg a h_{2}^{\prime}=h_{1}^{\prime}\right\| \rightarrow A_{g} \nsucceq B$ so, since $A_{g}$ is maximal in $B,\left\|\neg a h_{2}^{\prime}=h_{1}^{\prime}\right\| \rightarrow A_{g} \leq A_{g}$ and we are done.

(iii) $\widetilde{\varphi_{1} \wedge \varphi_{2}} \equiv \tilde{\varphi}_{1} \wedge \tilde{\varphi}_{2}$. For 1 . if $\mathbb{N}^{+} \models \varphi_{1} \wedge \varphi_{2}$, then $\mathbb{N}^{+} \models \varphi_{1}$ and $\mathbb{N}^{+} \models \varphi_{2}$. By the inductive hypothesis $\left\|\tilde{\varphi}_{1}\right\| \geq B$ and $\left\|\tilde{\varphi}_{2}\right\| \geq B$, the statement follows. For 2. if $\mathbb{N}^{+} \not \forall \varphi_{1} \wedge \varphi_{2}$ then $\mathbb{N}^{+} \not \forall \varphi_{1}$ or $\mathbb{N}^{+} \not \neq \varphi_{2}$. Let us assume that $\mathbb{N}^{+} \not \forall \varphi_{1}$. Then by the inductive hypothesis $\left\|\tilde{\varphi}_{1}\right\| \leq A_{g}$. From this $\|\tilde{\varphi}\| \leq A_{g}$. 3. again easily follows from the inductive hypothesis and the maximality of $A_{g}$. 
(iv) $\widetilde{\varphi_{1} \vee \varphi_{2}} \equiv \tilde{\varphi}_{1} \vee \tilde{\varphi}_{2}$. Similar to the previous case.

(v) $\widetilde{\neg \varphi_{1}} \equiv \tilde{\varphi}_{1} \rightarrow B(y)$. Let us assume that $\mathbb{N}^{+} \models \neg \varphi_{1}$. By the inductive hypothesis $\left\|\tilde{\varphi}_{1}\right\| \leq A_{g}$, so $\left\|\tilde{\varphi}_{1}\right\| \rightarrow A_{g}=\top \geq B$. If $\mathbb{N}^{+} \not \models \neg \varphi_{1}$, ie. $\mathbb{N}^{+} \models \varphi_{1}$, then $\left\|\tilde{\varphi}_{1}\right\| \geq B$. If $\left\|\tilde{\varphi}_{1}\right\| \rightarrow A_{g} \geq B$, then $B \leq\left(\left\|\tilde{\varphi}_{1}\right\| \wedge\left(\left\|\tilde{\varphi}_{1}\right\| \rightarrow A_{g}\right) \leq A_{g}\right.$, so $B \leq A_{g}$, a contradiction. From this $\left\|\tilde{\varphi}_{1}\right\| \rightarrow A_{g} \nsupseteq B$ and by the maximality of $A_{g},\left\|\tilde{\varphi}_{1}\right\| \rightarrow A_{g} \leq A_{g}$.

(vi) $\exists \widetilde{x \varphi_{1}(x)} \equiv \exists x\left(\psi_{\mathbb{N}^{+}}(x) \wedge \tilde{\varphi}_{1}(x)\right)$. Assume first that $\mathbb{N}^{+} \models \exists x \varphi_{1}(x)$. Then $\mathbb{N}^{+} \models$ $\varphi_{1}(a)$ for some $a \in \mathbb{N}^{+}$and $\left\|\psi_{\mathbb{N}^{+}}(a)\right\| \geq\|a=a\|=\top$. By the inductive hypothesis $\left\|\tilde{\varphi}_{1}(a)\right\| \geq B$, from these 1. follows. If $\mathbb{N}^{+} \not \forall \exists x \varphi_{1}(x)$, then for all $a \in \mathbb{N}^{+} \mathbb{N}^{+} \forall \varphi_{1}(a)$ and by the inductive hypothesis $\left\|\tilde{\varphi}_{1}(a)\right\| \leq A_{g}$. Let $h \in \mathcal{G}$ arbitrary. Then by Theorem 7. $\left\|\psi_{\mathbb{N}^{+}}(h)\right\|=\bigvee_{k \in \mathbb{N}^{+}}\|h=k\|$, so $\left\|\psi_{\mathbb{N}^{+}}(a) \wedge \tilde{\varphi}_{1}(a)\right\|=\bigvee_{k \in \mathbb{N}^{+}}\left(\|h=k\| \wedge\left\|\tilde{\varphi}_{1}(h)\right\|\right) \leq \bigvee_{k \in \mathbb{N}^{+}}\left(\left\|\tilde{\varphi}_{1}(k)\right\|\right) \leq A_{g}$ and 2. follows. For 3., since $A_{g}$ is maximal in $B$, it is enough to prove that $\left\|\exists x\left(\psi_{\mathbb{N}^{+}}(x) \wedge \tilde{\varphi}_{1}\left(x, h_{2}^{\prime}, g, h_{1}^{\prime}\right)\right)\right\| \geq B \Leftrightarrow\left\|\exists x\left(\psi_{\mathbb{N}^{+}}(x) \wedge \tilde{\varphi}_{1}\left(x, h_{2}^{W}, g, h_{1}\right)\right)\right\| \geq B$. If $\left\|\exists x\left(\psi_{\mathbb{N}^{+}}(x) \wedge \tilde{\varphi}_{1}\left(x, h_{2}^{\prime}, g, h_{1}^{\prime}\right)\right)\right\| \geq B$ then there is $u \in \mathcal{G}$ such that $\| \psi_{\mathbb{N}^{+}}(u) \wedge$ $\left.\tilde{\varphi}_{1}\left(u, h_{2}^{\prime}, g, h_{1}^{\prime}\right)\right) \| \geq B$. From this $\bigvee_{k \in \mathbb{N}^{+}}\left(\|u=k\| \wedge\left\|\tilde{\varphi}_{1}\left(u, h_{2}^{\prime}, g, h_{1}^{\prime}\right)\right\|\right) \geq B$ by Theorem 7 so for some $k \in \mathbb{N}^{+}\|u=k\| \wedge\left\|\tilde{\varphi}_{1}\left(u, h_{2}^{\prime}, g, h_{1}^{\prime}\right)\right\| \geq B$. From this by the inductive hypothesis $\|u=k\| \wedge\left\|\tilde{\varphi}_{1}\left(u, h_{2}^{W}, g, h_{1}\right)\right\| \geq B$ and $\left\|\exists x\left(\psi_{\mathbb{N}^{+}}(x) \wedge \tilde{\varphi}_{1}\left(x, h_{2}^{W}, g, h_{1}\right)\right)\right\| \geq B$ follows. The other direction is similar.

(vii) $\widetilde{\forall x \varphi_{1}(x)} \equiv \forall x\left(\psi_{\mathbb{N}^{+}}(x) \rightarrow \tilde{\varphi}_{1}(x)\right)$. Similar to the previous case.

(viii) $\exists \widetilde{S \varphi_{1}(S)} \equiv \widetilde{\varphi_{1}(S / \emptyset)} \vee \exists v_{S} \exists u\left(\tilde{\varphi}_{1}\left(v_{S}, u\right)\right)$.

Let us assume that $\mathbb{N}^{+}=\exists S \varphi_{1}(S)$, so $\mathbb{N}^{+}=\varphi_{1}(W)$ for some $W \subseteq \mathbb{N}^{+}$. If $W=\emptyset,\left\|\widetilde{\varphi_{1}(S / \emptyset)}\right\| \geq B$ by the inductive hypothesis. If $W \neq \emptyset$, by the inductive hypothesis $\left\|\tilde{\varphi}_{1}\left(h_{2}^{W}, h_{1}\right)\right\| \geq B$, so $\left\|\exists v_{S} \exists u \tilde{\varphi}_{1}\left(v_{S}, u\right)\right\| \geq B$, ie. 1. follows. If $\mathbb{N}^{+} \not \models \exists S \varphi_{1}(S)$ then for all $W \subseteq \mathbb{N}^{+}, \mathbb{N}^{+} \not \models \varphi_{1}(W)$, and $\left\|\tilde{\varphi}_{1}\left(h_{2}^{W}, h_{1}\right)\right\| \leq A_{g}$. For every pair $h_{2}^{\prime}, h_{1}^{\prime}$ in $\mathcal{G}$ we have to show that $\left\|\tilde{\varphi}_{1}\left(h_{2}^{\prime}, h_{1}^{\prime}\right)\right\| \leq A_{g}$. By 3. applied to $\varphi_{1}$, for some $W \subseteq \mathbb{N}^{+}\left\|\tilde{\varphi}_{1}\left(h_{2}^{\prime}, h_{1}^{\prime}\right)\right\| \leq A_{g}$ if and only if $\left\|\tilde{\varphi}_{1}\left(h_{2}^{W}, h_{1}\right)\right\| \leq A_{g}$ and 2. follows. Using the inductive hypothesis 3. is immediate, since $\exists S \varphi_{1}(S)$ does not contain $v_{s}$ and $u$ free.

(ix) $\forall \widetilde{S \varphi_{1}(S)} \equiv \forall v_{S} \forall u \tilde{\varphi}_{1}\left(v_{S}, u\right)$. Let us assume that $\mathbb{N}^{+} \models \forall S \varphi_{1}(S)$, so $\mathbb{N}^{+} \models$ $\varphi_{1}(W)$ for all $W \subseteq \mathbb{N}^{+}$. By the inductive hypothesis $\left\|\tilde{\varphi}_{1}\left(h_{2}^{W}, h_{1}\right)\right\| \geq B$. If $h_{1}^{\prime}, h_{2}^{\prime} \in \mathcal{G}$ are arbitrary, apply 3. to $\varphi_{1}$. There is some $W \subseteq \mathbb{N}^{+}$such that $\left\|\tilde{\varphi}_{1}\left(h_{2}^{\prime}, h_{1}^{\prime}\right)\right\| \geq B$ if and only if $\left\|\tilde{\varphi}_{1}\left(h_{2}^{W}, h_{1}\right)\right\| \geq B$. From these 1 . follows. If $\mathbb{N}^{+} \not \forall \forall S \varphi_{1}(S)$ then for some $W \subseteq \mathbb{N}^{+}, \mathbb{N}^{+} \not \forall \varphi_{1}(W)$, and $\left\|\tilde{\varphi}_{1}\left(h_{2}^{W}, h_{1}\right)\right\| \leq A_{g}$ 
by the inductive hypothesis. From this 2. follows. Again, using the inductive hypothesis, 3. is immediate.

Theorem 14. Let $\varphi$ be a second order $L^{\prime}$ sentence, $\psi \equiv \forall y(\zeta(y) \rightarrow \tilde{\varphi}(y))$.

1. If $\mathbb{N}^{+}=\varphi$, then $\|\psi\|=\top$.

2. If $\mathbb{N}^{+} \forall \varphi$, then $\|\psi\|=\perp$.

Proof. $\quad$ 1. If $\mathbb{N}^{+} \models \varphi$. Let $g \in \mathcal{G}$ arbitrary, we have to show that $C_{g} \leq\|\tilde{\varphi}[g]\|$. If $A_{g}=C_{g}$, then the statement follows from Lemma 12. By Lemma 13.1. $\|\tilde{\varphi}[g]\| \geq B$ for every $B$ with $A_{g}$ being maximal in $B$. By Lemma 11.1. if $A_{g}$ is maximal in $B$, then $B \leq C_{g}$, so we can apply Lemma 11.2. to get $\|\tilde{\varphi}[g]\| \geq \bigvee\left\{C \leq C_{g} \mid A_{g}\right.$ is maximal in $\left.C\right\}=C_{g}$.

2. If $\mathbb{N}^{+} \forall \varphi$, then by Lemma 13.2. for all $g \in \mathcal{G}\|\tilde{\varphi}[g]\| \leq A_{g}$. Let $\mathcal{G}_{0}=$ $\left\{g \in \mathcal{G} \mid A_{g}\right.$ is maximal $\}$. For any $h \in \mathcal{G}, g \in \mathcal{G}_{0}$, since $A_{g}$ is maximal, $A_{h} \vee A_{g}=A_{g}$, or $A_{h} \vee A_{g}=\top$. In the firs case $\left(A_{h} \leq A_{g}\right)$, so $\left(A_{h} \rightarrow A_{g}\right)=\top$, so $\left(A_{h} \rightarrow A_{g}\right) \vee A_{h}=\top$. In the second case $\left.\top=A_{h} \vee A_{g} \leq A_{h} \vee\left(A_{h} \rightarrow A_{g}\right)\right)$ so in both cases $\left(A_{h} \rightarrow A_{g}\right) \vee A_{h}=\top$. Since $h$ was an arbitrary element, if $A_{g}$ is maximal, $C_{g}=\top$ and $C_{g} \rightarrow A_{g}=A_{g}$ then, since $A_{g} \leq C_{g}$. So, using Lemma 11.2 and the fact that each $B \in \mathcal{H}_{1}$ is of the form $A_{g}$ for some $g \in \mathcal{G}$, $\|\psi\| \leq \bigwedge\left\{B \in \mathcal{H}_{1} \mid B\right.$ is maximal $\}=\perp$.

Theorem 15. True second-order arithmetic can be interpreted in the real algebraic structure of models of intuitionistic analysis built on nice Heyting algebras.

\section{References}

[1] M. Erdélyi-Szabó, Undecidability of the real-algebraic structure of models of intuitionistic elementary analysis, The Journal of Symbolic Logic 65, 1014-1030 (2000).

[2] M. Erdélyi-Szabó, Undecidability of the real-algebraic structure of Scott's model, Mathematical Logic Quarterly 44, 344-348 (1998).

[3] D. S. Scott, Extending the topological interpretation to intuitionistic analysis, Compositio Math. 20, 194-210 (1968). 
[4] P. Scowcroft, A new model for intuitionistic analysis, Ann. Pure Appl. Logic 47, 145-165 (1990).

[5] S. C. Kleene and R. E. Vesley, The Foundations of Intuitionistic Mathematics (North-Holland, Amsterdam, 1965).

[6] A. Heyting, Intuitionism. An Introduction (North-Holland, Amsterdam, 1956).

[7] P. Scowcroft, Some purely topological models for intuitionistic analysis, Ann. Pure Appl. Logic 98, 194-210 (1999).

[8] M. D. Krol, A topological model for intuitionistic analysis with Kripke's scheme, Z. Math. Logik Grundlag. Math. 24, 427-436 (1978). 